\title{
A catalogue of putative unique transcripts from Douglas-fir (Pseudotsuga menziesii) based on 454 transcriptome sequencing of genetically diverse, drought stressed seedlings
}

Thomas Müller ${ }^{1}$, Ingo Ensminger ${ }^{2,3^{*}}$ and Karl J Schmid ${ }^{1 *}$

\begin{abstract}
Background: Douglas-fir (Pseudotsuga menziesii) extends over a wide range of contrasting environmental conditions, reflecting substantial local adaptation. For this reason, it is an interesting model species to study plant adaptation and the effects of global climate change such as increased temperatures and significant periods of drought on individual trees and the forest landscape in general. However, genomic data and tools for studying genetic variation in natural populations to understand the genetic and physiological mechanisms of adaptation are currently missing for Douglas-fir. This study represents a first step towards characterizing the Douglas-fir transcriptome based on 454 sequencing of twelve cDNA libraries. The libraries were constructed from needle and wood tissue of coastal and interior provenances subjected to drought stress experiments.

Results: The 454 sequencing of twelve normalized cDNA libraries resulted in 3.6 million reads from which a set of 170,859 putative unique transcripts (PUTs) was assembled. Functional annotation by BLAST searches and Gene Ontology mapping showed that the composition of functional classes is very similar to other plant transcriptomes and demonstrated that a large fraction of the Douglas-fir transcriptome is tagged by the PUTs. Based on evolutionary conservation, we identified about 1,000 candidate genes related to drought stress. A total number of 187,653 single nucleotide polymorphisms (SNPs) were detected by three SNP detection tools. However, only 27,688 SNPs were identified by all three methods, indicating that SNP detection depends on the particular method used. The two alleles of about $60 \%$ of the 27,688 SNPs are segregating simultaneously in both coastal and interior provenances, which indicates a high proportion of ancestral shared polymorphisms or a high level of gene flow between these two ecologically and phenotypically different varieties.

Conclusions: We established a catalogue of PUTs and large SNP database for Douglas-fir. Both will serve as a useful resource for the further characterization of the genome and transcriptome of Douglas-fir and for the analysis of genetic variation using genotyping or resequencing methods.
\end{abstract}

*Correspondence: ingo.ensminger@utoronto.ca;

karl.schmid@uni-hohenheim.de

2 Department of Biology, University of Toronto at Mississauga, Mississauga,

ON, Canada

${ }^{1}$ Department of Crop Biodiversity And Breeding Informatics, University of

Hohenheim, Stuttgart, Germany

Full list of author information is available at the end of the article 


\section{Background}

Douglas-fir (Pseudotsuga menziesii (Mirb.) Franco) is an ecologically highly variable species that occurs in two main varieties in North America. The natural range of the coastal or green Douglas-fir (Pseudotsuga menziesii var. menziesii) extends over 2,200 km from the Pacific Northwestern fog belt and the adjacent summer-dry Coastal Range and Cascade mountains to the drier coastland of Southern California. The interior or blue Douglas-fir (Pseudotsuga menziesii var. glauca) is distributed over more than 4,500 $\mathrm{km}$ along the dry continental climates of the montane to the subalpine Rocky Mountains from Alberta to Colorado with isolated populations reaching into Mexico. Douglas-fir grows from sea level on Vancouver Island up to 3,000 m altitude in the southern Rocky Mountains [1]. Within its natural range, Douglas-fir has evolved into a variety of genetically diverse populations adapted to contrasting ecozones (e.g. [2,3]).

Douglas-fir populations differ in their response to frost [4,5], drought [5-7], and along environmental gradients $[4,8]$. Like most conifer species, it is able to cope with limitations in soil water availability within its natural range $[9,10]$. There is a negative relationship between shoot water potential and the photosynthesis rate [6], which decreased by about $70 \%$ in water-stressed trees with a predawn shoot water potential of about -1.7 MPa. In conifers such as Douglas-fir or pine, the recovery of photosynthesis upon rainfall and rewatering occurs within days together with the rapid recovery of predawn shoot water potential from stressed (around -1.5 MPa), or mildly stressed (around -1.0 $\mathrm{MPa}$ ) to values higher than $-0.5 \mathrm{MPa}[6,11]$. This high ecological, genetical and physiological diversity provides an excellent system to study the adaptation of conifer trees to contrasting environments.

Due to its rapid growth and favorable wood quality, Douglas-fir is an economically relevant species for timber production. In Europe, the area of Douglas-fir production is rising [12]. Forest practitioners appreciate the resistance of Douglas-fir against many European pathogens [13,14]. It is also expected that Douglas-fir is better adapted to future climate conditions in Central Europe than e.g. Norway spruce [15].

The Intergovernmental Panel on Climate Change (IPCC) expects increasing summer temperatures and decreasing precipitation in Central Europe in the coming years [16]. A trend towards warmer summers and more frequent summer droughts was reported in recent studies and simulations [17-19]. For this reason, it is important for forest managers to select suitable tree species or provenances that are adapted to the anticipated future climate. Currently, coastal Douglas-fir provenances are more frequently planted in Central Europe due to their superior growth performance compared to interior Douglasfirs $[13,14,20]$. The identification and characterization of differentially adapted provenances of coastal and interior Douglas-fir varieties has therefore high practical value.

Because of the large genome size of Douglas-firs (18.7 Gb, about 100 times the genome size of Arabidopsis thaliana [21,22] or 37 times the genome size of poplar $[23,24])$, transcriptome analysis is a cost-effective and suitable approach for the identification of candidate genes for adaptive traits and molecular markers that are linked to phenotypic variation. Transcriptomes of many species have been analyzed by next-generation sequencing technologies [25-27], and numerous coding single-nucleotide polymorphisms (SNPs) were identified in conifer species such as Pinus contorta, Picea glauca and Pinus taeda $[26,28,29]$.

Douglas-firs, like other forest trees, have a high level of genetic diversity [30,31]. For example, one study identified 933 SNPs in 121 candidate genes for cold-hardiness (1 SNP per $43 \mathrm{bp}$ to $1 \mathrm{SNP}$ per $112 \mathrm{bp}$ ) in coastal Douglasfirs [32]. For this reason, transcriptome sequencing of different provenances will lead to candidate genes for differential adaptation and to many new genetic markers for the characterization of different populations.

The purpose of this study was to establish a catalogue of Douglas-fir putative unique transcripts (PUTs) enriched for drought stressed genes and to identify genetic polymorphisms as resource for further analysis such as resequencing projects, association studies, and gene expression profiling.

\section{Results}

Sequencing and assembly

The sequencing of twelve cDNA libraries resulted in $3,619,544$ reads with an average length of $338 \mathrm{bp}$. After preprocessing, the number of reads decreased to $2,957,373$. Read numbers were not equally distributed among libraries (Additional file 1). The DINM, DINS and DIWC libraries consisted of less than 200,000 reads each and the DIWM library of less than 100,000 reads (see Table 1 for an explanation of the library abbreviations). The average length of the reads decreased to $315 \mathrm{bp}$ after pre-processing (Additional file 1). More than $99 \%$ of reads in each library were used for the construction of the assembly after quality trimming, with the exception of the

Table 1 Explanation of the cDNA library abbreviations

\begin{tabular}{lllll}
\hline \multirow{2}{*}{ Variety } & Tissue & Control & Mild stress & Severe stress \\
\cline { 3 - 5 } Costal & Needles & DCNC & DCNM & DCNS \\
& Wood & DCWC & DCWM & DCWS \\
\multirow{2}{*}{ Interior } & Needles & DINC & DINM & DINS \\
& Wood & DIWC & DIWM & DIWS
\end{tabular}

$\mathrm{D}=$ Douglas-fir, $\mathrm{C} / \mathrm{I}=$ coastal/interior, $\mathrm{N} / \mathrm{W}=$ needle/wood tissue, $\mathrm{C} / \mathrm{M} / \mathrm{S}=$ no/mild/severe drought stress. 
DIWM library (95\% used). A total of 2,793,051 (94.44\%) reads were assembled into 141,626 isotigs (of which 275 were contigs) of at least $100 \mathrm{bp}$ length. Additional file 2 contains the origin and the number of assembled reads. All isotigs were clustered in 116,311 isogroups. The mean isotig length was $623.22 \mathrm{bp}$ (s.d. $437.67 \mathrm{bp}$, median: 474 bp), the mean coverage per base was 5.0 reads (s.d. 8.07), and the mean number of reads per isotig was 44.5 (s.d. 145.54). For 21,837 isotigs longer than 999 bp, the mean coverage increased to 13.66 (s.d. 11.77) reads per base. Furthermore, the mean number of reads per isotig reached 181.27 (s.d. 274.75). Length of the isotig was positively correlated with the number of reads $(r=0.4972$, $P<0.0001$; Additional file 3).

Based on the results of the assembly, we constructed a set of PUTs as outlined in the Methods section. 42,159 of 71,392 reads with a length $>99$ bp initially labelled as singletons were mapped to isotigs and were considered as false positive singletons. Therefore, the final PUT set consisted of 170,859 sequences (141,626 isotigs and 29,233 singletons) with an average sequence length of $564.6 \mathrm{bp}$ (s.d. 420.86 bp, median: 431 bp, Additional file 4). As no reference sequence of Douglas-fir was available, we used the PUT set as reference for the following analysis, including functional annotation and SNP detection.

\section{Functional annotation of the PUTs}

For functional annotation, we compared all PUTs to the NCBI $n r$ database using BLASTX with an e-value cutoff of $e^{-10}$. At least one BLAST hit was obtained with 46,645 transcripts. If only the best hit of each transcript is considered, a total of 20,604 different sequences (unique hits) were hit in the $n r$ database. The largest number of hits was against Picea sitchensis sequences, followed by Vitis vinifera (Figure 1). In the subsequent analysis, Blast2GO assigned at least one GO term to 39,624 transcripts. For the three main GO categories, 34,660 transcripts were assigned a $\mathrm{GO}$ term from the molecular function category, 28,714 from the biological process, and 24,166 from the cellular component category. To compare the distribution of GO terms of the Douglas-fir transcriptome with the distribution of GO terms of transcriptomes from other species, we also applied Blast2GO to the Arabidopsis thaliana and Picea sitchensis sequences downloaded from TAIR and NCBI, respectively. We chose these two species for comparison because $A$. thaliana is a well studied model species with a well studied transcriptome and $P$. sitchensis is the species with most top BLASTX hits in our Douglas-fir PUT set. Figure 2 and Additional file 5 show that the distributions of GO terms at GO level 2 to 5 for each of the three ontology classes are highly similar for all three species.

\section{Identification of treatment-specific PUTs}

The isotigs (non-singleton transcripts of the PUT set) were divided and clustered according to the origin of their reads (Figure 3). About one third (34.38\%) of the isotigs contained reads from all three treatments and were therefore grouped in the cms group. The average length of isotigs in the cms group was 995.32 bp (Table 2). Each of the $c m, c s$, and $m s$ groups contained $6-8 \%$ of the isotigs with an average isotig length of 489 to $496 \mathrm{bp}$. The $c$,

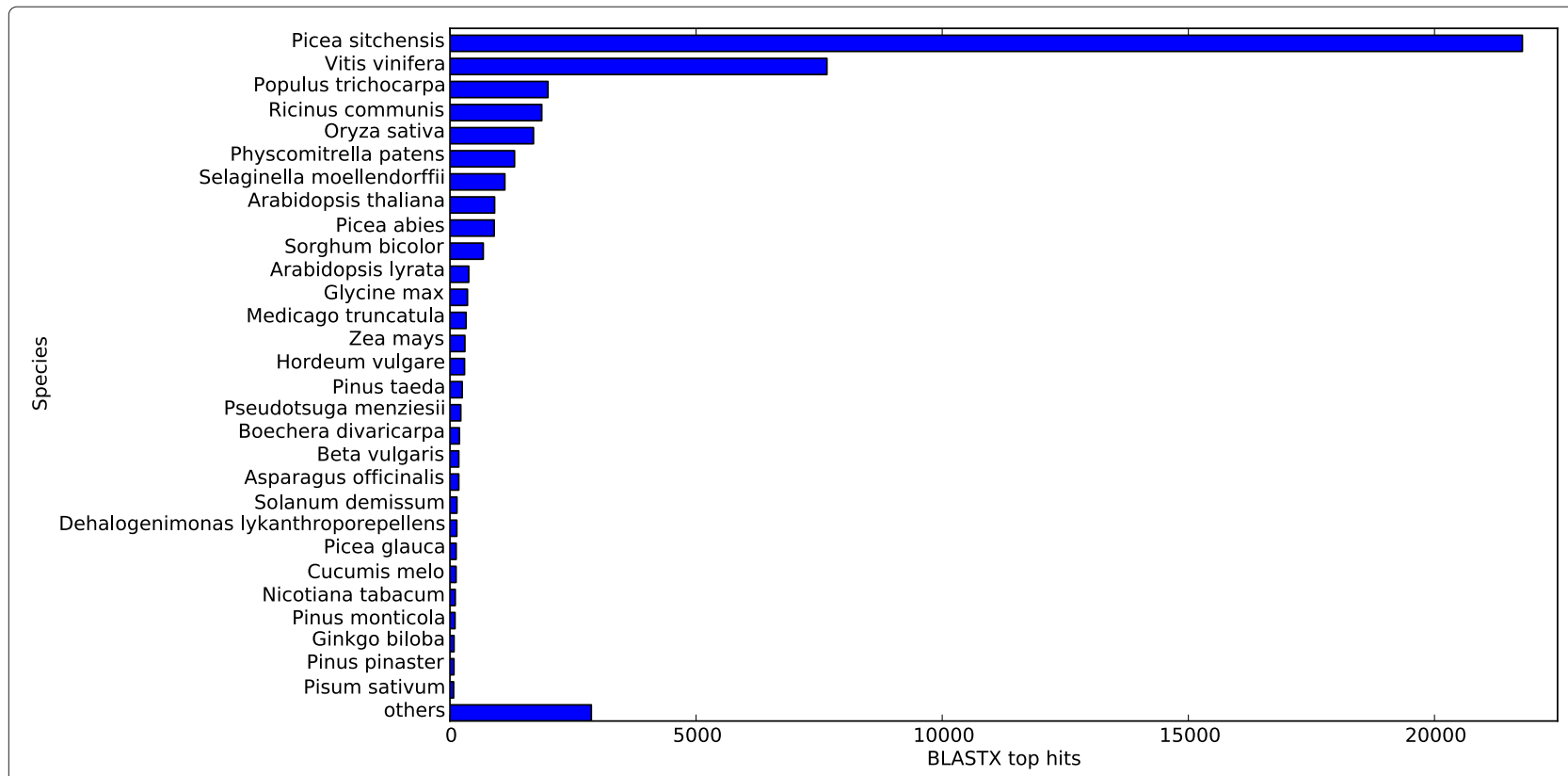

Figure 1 Distribution of the top hits species of the BLASTX search of the PUTs from the assembly against NCBI's $n r$ database. 


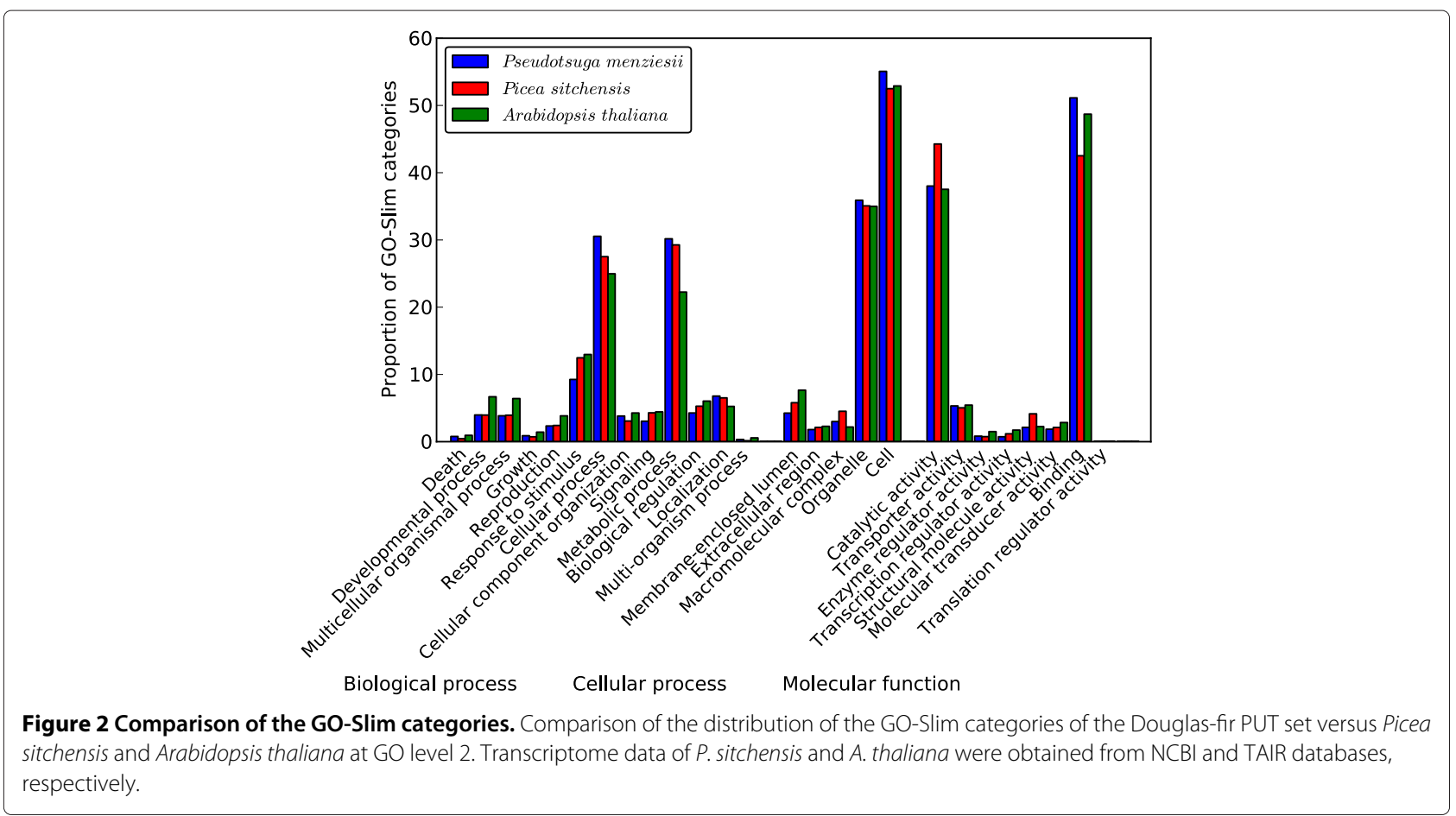

$m$, and $s$ groups contained $14-15 \%$ of the isotigs in each case. The average lengths of those isotigs were between 393 and 405 bp. The search for specific keywords in the BLASTX results revealed that 1,503 different isotigs coming from 998 isogroups had a BLASTX hit containing one of the keywords related to stress (Table 3, Additional file 6, Additional file 7). 134 of those isotigs coming from 132 isogroups were part of the $m, s$, or $m s$ groups and will serve as top candidate genes in future studies. We expected that cms group sequences are more conserved than sequences assigned to the remaining groups because drought stress specific sequences may evolve faster or are of a more recent evolutionary origin than common or widely expressed genes.

To test this hypothesis, we determined the proportion of significant BLASTX hits within each group of isotigs against the $n r$, the ara, and the picea databases (Table 2). Most hits were observed in the cms group (e.g. $58.11 \%$ against $n r$ ) and the least number of hits in the $m$ group (14.13\% against $n r)$. However, there is a highly significant correlation between the average length of isotigs and percent BLAST hits (e.g. hits against ara, $P<0.0001$, Table 4), and also between the total sequence length of each isotig group with the proportion of BLAST hits (e.g. hits against ara, $P=0.003$ ). Hence, the differences in the proportion of BLAST hits among classes of isotigs are not a result of differential evolutionary conservation, but of the amount of sequence data in each class.

\section{SNP identification}

SNP detection was performed with three different programs, GSMapper, ssahaSNP, and bwa/SAMtools, to minimize the number of false positives. PUTs obtained from the assembly served as reference. The programs detected 57,691 (Newbler), 155,269 (ssahaSNP), and 85,346 (bwa/SAMtools) SNPs, resulting in a total number of 187,653 different SNPs. However, only 27,688 SNPs were detected by all three tools (Figure 4). These SNPs were selected for further analysis because we consider them as most reliable true positive polymorphisms. These SNPs were distributed over 10,517 different PUTs of 10,054 different isogroups. Most transcripts harbored only a single SNP and 2,499 transcripts contained more than three SNPs. A total of 23 SNPs were detected in the most polymorphic PUT. In the 7,684 transcripts with at least one SNP and a significant match against the $n r$ database, 5,378 SNPs were classified as synonymous and 4,129 as non-synonymous.

In addition, we estimated the polymorphism level of the transcriptome by dividing the number of SNPs with the total number of different nucleotides in PUTs (as the same contig can contribute to several isotigs, see Additional file 8). If only the most reliable SNPs are considered, the estimated nucleotide diversity $(0.04 \%$, corresponding to approximately 1 SNP per 2,530 nucleotides) is very low. Using all SNPs identified by Newbler, bwa/SAMtools and ssahaSNP separately, resulted in estimated polymorphism levels of $0.08 \%$ (1 SNP per $1213 \mathrm{bp}$ ), $0.12 \%$ 


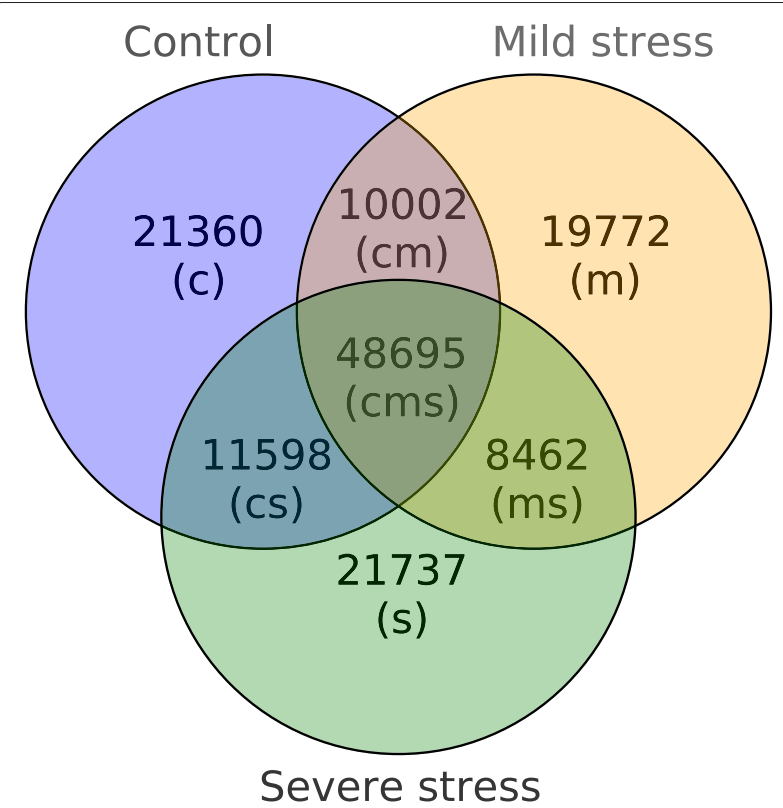

Figure 3 Composition of isotigs. Venn diagram showing the number of non-singleton PUTs (i.e. isotigs) consisting of reads from (one or several) libraries of one or more treatment(s). E.g. 21,737 isotigs are composed of reads originating from one or several of the severe stress libraries (DCNS, DINS, DCWS, DIWS). $c=$ control, $m=$ mild stress, $s=$ severe stress.

(1 SNP per $820 \mathrm{bp}$ ), and $0.22 \%$ (1 SNP per $451 \mathrm{bp}$ ), respectively.

To investigate differences in the level of genetic diversity between coastal and interior Douglas-firs, we divided the SNPs into groups depending on whether their alleles segregated in coastal or interior provenances, or in both (Table 5). The majority of SNPs are polymorphic in both coastal and interior provenances (Table 6), but coastal provenances have a higher number of provenance-specific alleles, as seen in the comparison of ci/c (7,158 SNPs) versus ci/i (2,547 SNPs) groups.

Table 2 Percentages of isotigs with BLASTX hits

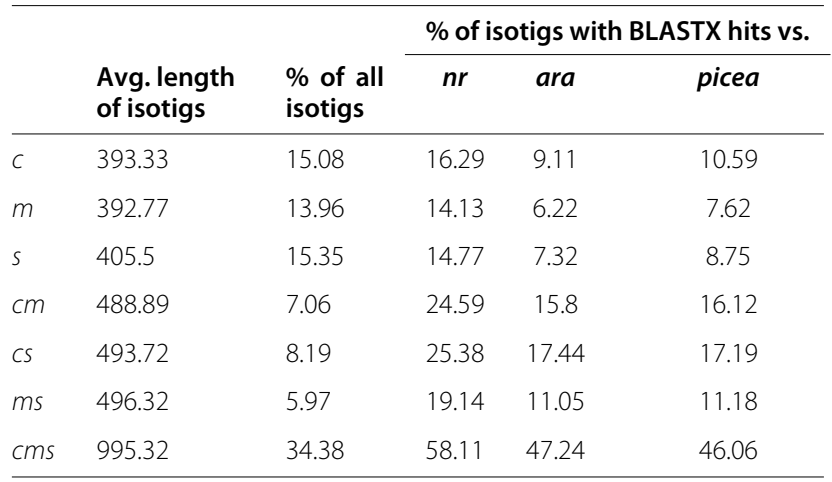

Percentages of isotigs (non-singleton PUTs) with a BLASTX hit against the $n r$, ara, and picea databases within the groups (see Figure 3 ). $c=$ control, $m=$ mild stress, $s=$ severe stress, $\mathrm{cm}=$ control and mild stress, $c s=$ control and severe stress, $m s=$ mild and severe stress, $\mathrm{cms}=$ control, mild and severe stress.
Table 3 Keyword search in BLASTX results

\begin{tabular}{|c|c|c|c|c|c|c|c|}
\hline \multirow[b]{2}{*}{ Search term } & \multicolumn{7}{|c|}{ Isotig group } \\
\hline & c & $m$ & $s$ & $\mathrm{~cm}$ & cs & $m s$ & $\mathrm{cms}$ \\
\hline "Drought" & 4 & 3 & 8 & 5 & 13 & 3 & 103 \\
\hline "Water-deficit" & 0 & 0 & 4 & 0 & 5 & 0 & 29 \\
\hline "Water-stress" & 6 & 2 & 6 & 4 & 6 & 1 & 109 \\
\hline "Osmotic-stress" & 4 & 1 & 6 & 2 & 7 & 2 & 58 \\
\hline "Heat-stress" & 2 & 0 & 1 & 0 & 2 & 0 & 27 \\
\hline "Heat-shock" & 24 & 17 & 31 & 21 & 23 & 15 & 466 \\
\hline "Dehydration" & 20 & 7 & 17 & 14 & 18 & 1 & 205 \\
\hline "Abscisic acid" & 7 & 1 & 8 & 5 & 10 & 2 & 142 \\
\hline "ABA-responsive"1 & 0 & 0 & 2 & 1 & 1 & 1 & 25 \\
\hline "ABA-induced" & 1 & 0 & 2 & 2 & 1 & 0 & 27 \\
\hline "ABA receptor" & 0 & 0 & 2 & 0 & 0 & 0 & 20 \\
\hline $\begin{array}{l}\text { "Pyrabactin } \\
\text { resistance 1" }\end{array}$ & 0 & 0 & 2 & 0 & 0 & 0 & 10 \\
\hline $\begin{array}{l}\text { "Snf1-related } \\
\text { protein kinases"2 }\end{array}$ & 4 & 2 & 2 & 2 & 3 & 7 & 69 \\
\hline "DREB1" & 2 & 0 & 0 & 1 & 1 & 0 & 9 \\
\hline "DREB2" & 2 & 0 & 0 & 2 & 2 & 0 & 14 \\
\hline "C-repeat binding" & 0 & 0 & 0 & 1 & 1 & 0 & 4 \\
\hline "ERD"4 & 7 & 4 & 9 & 3 & 8 & 2 & 112 \\
\hline "CIPK"5 & 2 & 3 & 2 & 0 & 2 & 6 & 47 \\
\hline "CDPK" & 0 & 2 & 1 & 7 & 1 & 0 & 39 \\
\hline "CBL1" 7 & 5 & 1 & 0 & 3 & 6 & 1 & 72 \\
\hline "PKS3" & 0 & 2 & 0 & 0 & 2 & 0 & 12 \\
\hline Different isotigs & 66 & 33 & 69 & 58 & 71 & 32 & 1,174 \\
\hline
\end{tabular}

Number of isotigs (non-singleton PUTs) with a BLASTX hit containing a keyword for each group (see Figure 3). $c=$ control, $m=$ mild stress, $s=$ severe stress,

$\mathrm{cm}=$ control and mild stress, $c s=$ control and severe stress, $m s=$ mild and severe stress, $\mathrm{cms}=$ control, mild and severe stress.

${ }^{1} \mathrm{ABA}=$ abscisic acid.

${ }^{2} \mathrm{Snf}=$ sucrose non-fermenting.

${ }^{3} \mathrm{DREB}=$ dehydration-responsive element-binding.

${ }^{4} E R D=$ early responsive to dehydration.

${ }^{5} \mathrm{CIPK}=\mathrm{CBL}$-interacting protein kinase.

${ }^{6} \mathrm{CDPK}=$ calcium-dependent protein kinase.

${ }^{7} \mathrm{CBL}=$ calcineurin $\mathrm{B}$-like protein.

${ }^{8} \mathrm{PKS}=$ phytochrome kinase substrate.

\section{Discussion}

\section{Sequencing and assembly}

Next generation sequencing (NGS) has now a major impact on the genome-wide analysis of transcriptomes in non-model species [25-27]. To achieve a comprehensive characterization of the protein-coding genome of Douglas-fir, we exposed young seedlings from different provenances to drought stress treatments and generated normalized cDNA libraries to enrich for rare transcripts or genes not constitutively expressed. All libraries were assembled into a single assembly to maximize the number of reads per transcript and to improve the quality of assembly and SNP annotation. There is a strong relationship between the number of reads and the length 
Table 4 BLASTX and Blast2GO results divided by isotig length

\begin{tabular}{|c|c|c|c|c|c|}
\hline & All PUTs & $<501$ & $501-1,000$ & $1,001-1,999$ & $>2,000$ \\
\hline Number of PUTs & 170,859 & 106,296 & 42,760 & 19,589 & 2,214 \\
\hline Total sequence [Mbp] & 96.5 & 35 & 26.4 & 29.7 & 5.4 \\
\hline Hits with $n r$ & $27.3 \%$ & $13.5 \%$ & $36.9 \%$ & $75.5 \%$ & $87.8 \%$ \\
\hline Hits with ara & $19.6 \%$ & $7.9 \%$ & $25.1 \%$ & $64.4 \%$ & $82.6 \%$ \\
\hline Hits with picea & $19.6 \%$ & $8.5 \%$ & $26.3 \%$ & $58.7 \%$ & $71.4 \%$ \\
\hline Isotigs with assigned GO term & $23.2 \%$ & $11.9 \%$ & $26.6 \%$ & $63.9 \%$ & $82 \%$ \\
\hline
\end{tabular}

Results of similarity searches with BLASTX and functional annotation using Blast2GO subdivided by transcript length in bp.

of a transcript, confirming the observation that longer transcripts consist of more reads than shorter transcripts [33]. The number and average length of the reads of four libraries (DINM, DINS, DIWC, and DIWM) were below expectation $[26,34,35]$, probably because of problems during the sequencing process. However, we did not exclude these libraries, because they contributed the same proportion of reads to the assembly as the other libraries (>95\% of the reads of each library).

94.44\% of all reads were assembled into isotigs during assembly, which is a high proportion compared to similar 454 transcriptome assemblies. For example, $88 \%$ of reads were assembled in Melitaea cinxia [36] and Eucalyptus grandis [25], 78\% in Pandinus imperator [37], and $48 \%$ in Pinus contorta [26]. One cause for the high proportion in our data is the stringent preprocessing of reads,

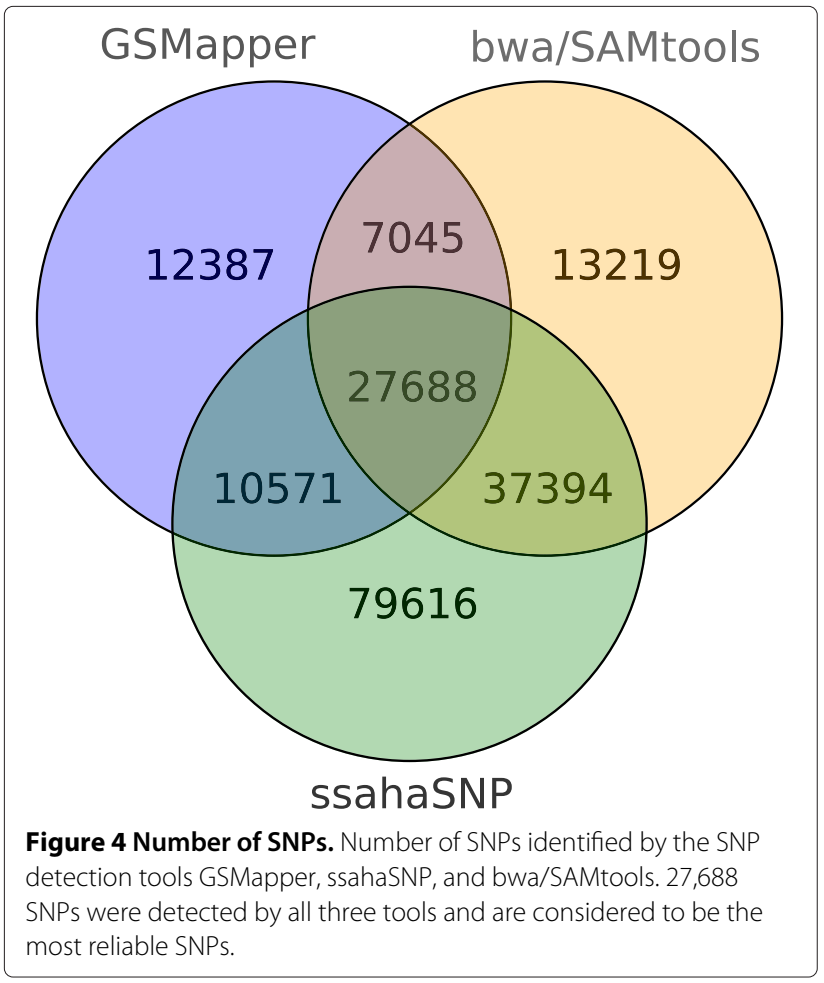

which excluded most uninformative reads prior to the assembly. The number of PUTs in the assembly $(170,859)$ exceeds the number of expected genes in conifer genomes, which ranges from 30.000 to 50.000 genes [38]. Nevertheless, the PUT set is smaller than the one obtained with Pinus contorta (303,450 transcripts) [26], but larger than in other 454 transcriptome sequencing projects (e.g. 44,469 transcripts in waterhemp [39], 108,297 transcripts in a butterfly species [36]). It should be noted that it is difficult to compare numbers of transcripts in different projects, because they are influenced by the genome and transcriptome sizes of the sequenced organism, the assembly method used [34], and the number of reads used for the assembly. Overall, the high number of transcripts compared to the expected number of genes is likely a result of incompletely assembled genes.

The average length of transcripts is 564.60 bp (median $431 \mathrm{bp}$, Additional file 4), which is approximately half of the expected average gene length in eukaryotes (1346 bp) [40].

\section{Functional annotation of PUTs}

We annotated the PUTs by using stringent BLASTX searches against the $n r$ database from NCBI with a cutoff e-value of $e^{-10}$. Assuming that each unique blast hit represents a different transcribed gene, we tagged 20,604 genes of the Douglas-fir genome. This number is similar to other projects in Pinus contorta with 17,321 tagged genes [26] and is lower than the estimated total number of about 30,000 transcribed genes in white spruce Picea glauca [38]. If we further assume that the number of unique blast hits equals the number of transcribed genes and that the transcriptome sizes of Douglas-fir and white spruce are comparable, it seems that the PUT set generated in this study tags about two thirds of the protein-coding genes of Douglas-fir. The missing third likely comprise (1) cDNAs that were excluded from assembly because of low quality; (2) genes that are expressed at different developmental stages, growth conditions, or tissues and were thus not represented in the twelve cDNA libraries despite the normalization process; and (3) non-conserved genes, which are either lineage-specific and not yet 
Table 5 Groups of SNPs

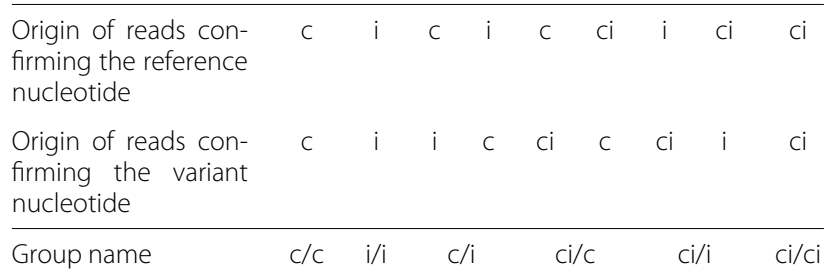

Partitioning of SNPs into groups depending on the origin (coastal vs. interior) of sequence reads. c: only reads of coastal libraries; i: only reads of interior libraries, ci: reads of coastal as well as interior libraries; ci/ci: both possible nucleotides were confirmed by reads of coastal and interior libraries; $\mathrm{c} / \mathrm{i}$ : one of the possible nucleotides at the SNP position was confirmed only by reads of coastal libraries, the other nucleotide only by reads of interior libraries; etc.

present in the $n r$ database or rapidly evolving genes with e-values $>e^{-10}$ in the BLASTX comparisons. Since about $75 \%$ of the transcripts do not result in significant BLASTX hits, one may speculate that a large proportion represents non-conserved genes.

Gene Ontologies (GOs) provide a standardized set of terms to describe genes and gene products consistently in different species and databases [41]. GO terms are widely used for annotation and for comparisons of gene products of different species (e.g. $[26,42])$. The similarity of the GO annotation distributions of Douglas-fir PUTs to the well-characterized transcriptome of $A$. thaliana and the protein sequences of $P$. sitchensis (Figure 2) suggests that the PUT set broadly represents the Douglas-fir transcriptome and can be viewed as being representative for further applications and investigations.

\section{Identification of drought stress related genes}

Dividing PUTs consisting of multiple reads (i.e. the isotigs) by the origin of their reads is a simple, but useful method to identify potential treatment-specific sequences. About $50 \%$ of isotigs consist of reads from the single treatment groups $c, m$, or $s$. On average, they are shorter

Table 6 Summarized number of SNPs

\begin{tabular}{lc}
\hline Origin of reads at SNP position & Number of SNPs \\
\hline $\mathrm{Ci} / \mathrm{Ci}$ & 15,843 \\
$\mathrm{Ci} / \mathrm{C}$ & 7,158 \\
$\mathrm{Ci} / \mathrm{i}$ & 2,547 \\
$\mathrm{c} / \mathrm{i}$ & 886 \\
$\mathrm{C} / \mathrm{C}$ & 817 \\
$\mathrm{i} / \mathrm{i}$ & 437 \\
\hline
\end{tabular}

Number of SNPs with a specific composition of reads. ci/ci: variant and reference nucleotide appeared in reads from coastal and interior libraries; ci/c, ci/i: variant or reference nucleotide appeared only in reads of the coastal or interior libraries, the other one appeared in reads of both kind of libraries; c/i: variant or reference nucleotide appeared only in reads of the coastal libraries, the other one only in reads of the interior libraries; $c / c$, i/i: variant and reference nucleotides appeared only in reads of coastal or interior libraries. than isotigs containing reads from at least two different treatments. The isotigs of the $m$ and $s$ groups, but also the $m s$ group, were considered to be top candidates for drought stress tolerance or resistance. However, since most treatment-specific isotigs consist of only two or three reads that originated from a total of twelve cDNA libraries, we reasoned that the power of a statistical model to detect significant differences is low. Therefore, we compared the extent of evolutionary conservation between groups as judged by the proportion of significant BLAST hits. This analysis is based on the notion that widely expressed genes are under stronger selective constraint than treatment-specific genes $[43,44]$. Under the assumption that constitutively expressed genes are expressed in all different treatments, we expected that $\mathrm{cms}$ group isotigs are more conserved than isotigs from the $c, m$, and $s$ groups. Since the libraries were normalized and cDNA levels do not represent true expression levels, we restricted our analysis to presence-absence patterns. The differences in the fractions of BLASTX hits in single treatment groups and the cms group suggested that genes expressed in all three treatments are more conserved. However, if groups are corrected for the total amount of sequence data, $\mathrm{cms}$ group isotigs are not more conserved than treatment-specific isotigs, because the main determinant for a BLAST hit is isotig length which is longer in $\mathrm{cms}$ isotigs (Table 4). This pattern was also observed in white spruce [38].

In addition to testing the general hypothesis that treatment-specific genes are less conserved than widelyexpressed genes, we also parsed BLASTX results for drought stress related keywords to find potential drought stress related PUTs. We expect that the 1,503 transcripts with a BLASTX hit containing one of the keywords are probably involved in the Douglas-firs response to drought (Table 3). More than 1,100 candidate PUTs are part of the cms group and only 134 candidates are part of the $m, s$, and $m s$ groups. This reflects that the response to drought seems to be mainly facilitated through changes in gene expression levels via up- or down-regulation. The small set of 134 PUTs exclusively induced by drought stress appears to contribute to a specific drought response, but this needs to be further verified because their expression pattern may reflect a sampling artifact. Even though the function of those PUTs may not be conserved across large evolutionary distances, the identified PUTs serve as top candidates for further analysis of sequence and expression variation in comparisons of differentially adapted (e.g. coastal and interior) Douglas-fir provenances.

\section{Analysis of genetic variation}

The construction of the cDNA libraries representing genetically different provenances allowed the detection of 
SNPs for later analysis of patterns of genetic variation. The two most important results are the high proportion of shared polymorphisms and the strong influence of the SNP calling algorithm on the number of detected SNPs. By taking a conservative approach and considering only those SNPs that were called by all three programs, only 27,688 (highly reliable) SNPs were obtained, which is only about half of the number detected with gsMapper, which identified the lowest number of SNPs $(57,691)$. Since the numbers of called SNPs differed highly between SNP detection tools, our results indicate that SNP calling from next generation sequencing data depend to a high degree on the software tools used. Therefore, results should be interpreted with caution, if relying on a single SNP detection approach only. To our knowledge there are no systematic studies yet that compared the accuracy of different SNP callers with next-generation sequencing data.

A comparison of the SNP density (SNPs per $\mathrm{kb}$ ) of the most reliable SNPs with published data shows that the former is an underestimate of the true level of sequence variation in Douglas-fir. The SNP density is 1 SNP for every 2,530 bp, whereas other studies estimated an average SNP density from $1 \mathrm{SNP}$ per $43 \mathrm{bp}$ to $1 \mathrm{SNP}$ per 112 bp using Sanger sequencing protocols [32]. The reasons for the large difference to the reported SNP density are probably the stringency criteria used and the better quality of base-calling using Sanger sequencing. If we take only the SNPs identified by bwa/SAMtools or ssahaSNP in account, the SNP density increases to 1 SNP per $820 \mathrm{bp}$ and 1 SNP per $451 \mathrm{bp}$, respectively.

Nevertheless, our sequence data make a significant contribution to the number of Douglas-fir SNPs available for further applications. Until now, only around 1,300 SNPs have been identified in Douglas-fir [32,45]. If only the most reliable SNPs are considered, a key result is the large number of SNPs whose alleles are segregating in both the coastal and interior provenances $(15,483$ SNPs, ci/ci category in Table 6). In only $5 \%$ of SNPs $(886, \mathrm{c} / \mathrm{i})$ the two alleles are specific to coastal and interior provenances, respectively. This high proportion of shared polymorphisms indicates either a high level of shared ancestral polymorphisms between the two main Douglas-fir varieties, or recent, possibly pollen-mediated gene flow. The comparison of SNPs, in which only one of the two alleles is shared between coastal and interior provenances suggest a higher level of genetic diversity in coastal provenances because three times as many SNPs are polymorphic for both alleles in the coastal $(7,158$ SNPs in the ci/c group) than in the interior accessions $(2,547$ SNPs in the ci/i group). This difference is also observed for SNPs which were called only in either the interior or coastal provenances because no reads were available from the other provenance, respectively
(817 SNPs in the c/c versus 473 SNPs in the $\mathrm{i} / \mathrm{i}$ group). Although these results are consistent with earlier studies on the genetic diversity of Douglas-fir varieties[46,47], they are also certainly influenced by the different numbers of reads originating from coastal and interior cDNA libraries $(1,757,542$ vs. $1,076,192)$. Since there are $70 \%$ more reads from the coastal provenances, the probability of finding a polymorphism in these provenances is increased and needs to be accounted for in further conclusions.

Different numbers of reads can be accounted for by using methods for population genetic inference developed for next-generation sequencing that account for differences in read numbers from individuals or pools of individuals in estimating allele frequencies and population parameters $[48,49]$. However, such an approach does not work in the present study because allele frequencies depend on the sampled individuals in a library, the gene expression level and the effect of normalization on read numbers. Unbiased population genetic estimators like Tajima's $\pi$ can be calculated from 454 data [50], but as the coverage at most SNP positions is much smaller than the total number of individuals, the results are not reliable. The development of genotyping and resequencing arrays using the present set of PUTs to estimate SNP allele frequencies and population genetic inference will allow accurate and unbiased estimates of nucleotide diversity.

\section{Conclusions}

In this study we established a catalogue of Douglas-fir putative unique transcripts (PUTs) enriched for drought stress induced genes. Although the real magnitude of the transcriptome is yet unknown, we estimate that the majority of the transcriptome has been tagged by the PUT set presented here. This is based on the results of the functional annotation and the comparison of the GO term distributions with those of Arabidopsis thaliana and Picea sitchensis. By analyzing sequence variation in the PUTs we detected thousands of new SNPs. Furthermore, we identified drought stress specific candidate sequences. Taken together these data represent a useful resource for the next steps in the characterization of the Douglas-fir genome and transcriptome and the association of genetic variation with phenotypic traits such as adaptation to different ecogeographic environments.

\section{Methods}

\section{Plant material and library preparation}

1.5 year old Douglas-fir seedlings were obtained from tree nurseries in British Colombia (Canada), Washington, Colorado, and New Mexico (USA) and grown in the greenhouse in a mixture of soil:perlit:sand (50:25:25). All seedlings were fertilized with Osmocote Exact $\mathrm{Hi}$ End 5-6m (Scotts International BV, Heerlen, NL). Potted 
seedlings were watered every second day. Drought stress experiments started after two month of growth in the greenhouse, when visual inspection of the seedlings indicated a well developed root system. For the experiments, seedlings were randomly assigned to one of three different treatments: (1) control seedlings kept under well watered conditions, (2) mildly water stressed seedlings (predawn water potential between -0.7 and $-1.0 \mathrm{MPa}$ ) and (3) severely water stressed seedlings (predawn water potential between -1.5 and $-2.0 \mathrm{MPa}$ ). Water stress was imposed by withholding watering until a desired water potential had been reached [11]. Water potential was assessed by repeated measurements of predawn needle water potential using a Scholander pressure chamber to assess the level of water stress [51]. Within about 3-4 and 5-6 weeks, the target water potential was observed in the mildly and severely water stressed seedlings, respectively, and needles and sections of the stem (wood tissue) were harvested. Tissue from control seedlings was harvested in parallel in order to obtain samples from similarly aged plant material. Tissue samples were immediately frozen in liquid nitrogen and stored at $-80^{\circ} \mathrm{C}$ for later extraction of RNA.

Frozen needles or sections of the stem were homogenized using mortars and pistils chilled with liquid nitrogen until a fine powder was obtained. Total RNA was extracted from $300 \mathrm{mg}$ aliquots of the frozen powder using the CTAB method [52]. Isolated RNA from individual seedlings was then quality checked using Qiaxcel (Qiagen, Hilden, Germany).

Aliquots of the RNA from several seedlings and several provenances were then combined for synthesis into a total of twelve pooled RNA samples. Six of these pooled samples represented a subset of coastal and six samples represented a subset of interior Douglas-fir. Each of these two subsets included two sets of pooled RNA samples from either needle tissue or from wood tissue. Finally, each of these tissue specific sets consisted of one pooled RNA sample from control, mildly water stressed or severely water stressed seedlings (Table 1, Additional file 9).

Normalized cDNA libraries were generated by Evrogen LAB (Moscow, Russia). Starting from $0.3 \mu \mathrm{g}$ of total RNA double-stranded cDNA was synthesized using SMART Oligo II oligonucleotides and CDS primers (SMART Oligo II 5' -AAGCAGTGGTATCAA CGCAGAGTACGCrGrGrG- 3', CDS primer 5' AAGCAGTGGTATCAACGCAGAGTA-d(T)30[53]. Amplified cDNA was then purified using the QIAquick PCR purification kit (Qiagen, CA, USA), concentrated by ethanol precipitation and then diluted to a final cDNA concentration of $50 \mathrm{ng} / \mu \mathrm{l}$. SMART amplified cDNAs were then normalized [54]. Normalization included a cDNA denaturation/reassociation step followed by treatment with duplex-specific nuclease (DSN, [55]) and subsequent amplification of the normalized fraction by PCR using SMART PCR primers (SMART PCR primer 5' -AAGCAGTGGTAT CAACGCAGAGT-3').

454 sequencing of the normalized cDNA libraries was carried out by Seq-IT (Kaiserslautern, Germany) using a Genome Analyzer FLX with 454 titanium chemistry (Roche, Basel, Switzerland). Prior to sequencing, each cDNA library was first fragmented. Fragments were tagged with multiplex identifiers (MIDs) to allow library identification of the reads obtained from parallel sequencing of the libraries on the Genome Analyzer FLX. In total three titanium runs were performed, with 1.5 runs analyzing the needle libraries, and 1.5 runs analyzing the wood libraries. The proprietary genome analyzer software was used for the first preprocessing of sequence reads including the assignment of quality scores to generate .sff-files for further processing and assembly of the data.

\section{Preprocessing}

The resulting .sff-files were extracted with the sff_extract tool [56]. All sequences with at least one ' $N$ ' were removed. The preprocessed files were used as input for SnoWhite (release 1.1.3) [57], a cleaning pipeline for cDNA sequences that uses SeqClean [58] and trims polyA/T. All sequences shorter than $50 \mathrm{bp}$ or with a polyA/T repeat of at least $8 \mathrm{bp}$ at either end were discarded. The longer part of the sequence was retained if internal polyA/T tracts were detected. As the assembly program operates in flowgram signal space it is recommended to use .sff-files as input. Thus, the original .sff-files were altered according to the changes made during the preprocessing steps using custom Python scripts. Those altered .sff-files were loaded into the assembler.

\section{Assembly and mapping}

Sequences were assembled with Newbler v2.6 using default parameters supplemented by the -cdna and - urt options [34,59]. Newbler constructs a set of contigs (contiguous sequences), representing assembled reads. Unassembled reads were marked as singletons, repeats, outlier (e.g. chimeric reads), or too short. Isotigs consist of contigs connected by a subset of reads (Additional file 8). An isogroup is a group of different isotigs of the same multiple alignment. Isogroups represent genes, isotigs correspond to alternatively spliced transcripts, and contigs correspond to exons. This is a simplified view because contigs and isotigs can also contain sequences of untranslated regions. Independent contigs that were not part of an isotig were simply considered as isotigs to facilitate the analysis.

All twelve libraries were assembled together. Based on the assembly, we created a set of PUTs. We first searched 
for false positive singletons, i.e. reads that were marked as singletons although they matched nearly perfect to an existing isotig. For this purpose, all reads marked as singletons were mapped to the isotigs of the assembly using ssaha2 [60] with default parameter settings. Reads were mapped only if the pairwise sequence identity with a reference isotig was at least $98 \%$ of the alignment length. Unmapped reads were considered as real singletons and checked for duplicates. The final PUT set consisted of the isotigs and the singletons of the assembly representing all different transcripts found in the dataset. In particular, i.e. PUTs can be the only possible transcript of a gene, only a part of a longer transcript that can not be found within the data, alternatively spliced variants of a gene, but also the product of misassemblies. Sequences shorter than $100 \mathrm{bp}$ were excluded to dismiss potentially uninformative sequences.

\section{SNP detection}

SNPs were identified with GSMapper [59], ssahaSNP [60], and bwa/SAMtools [61,62]. Each program detected a different number of SNPs. Therefore, we combined the results of the three programs and considered the SNPs identified by all three tools as a set of potentially most reliable SNPs (Figure 5). We used the sequences of the PUTs derived from the assembly as reference for the SNP detection. To avoid sequencing errors from being considered as SNPs, we required for each tool that the reference

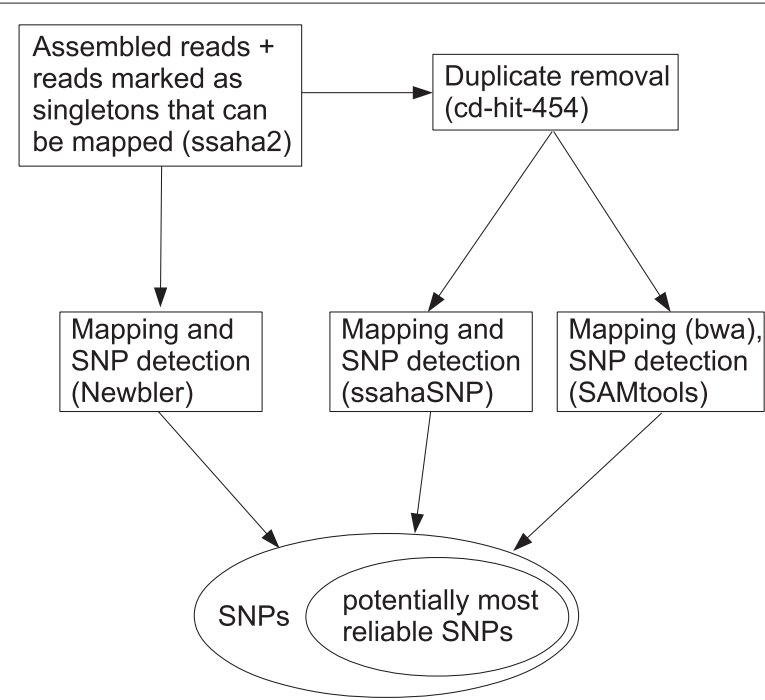

Figure 5 Workflow of the SNP detection. All reads that were assembled and all reads that were marked as singletons but that can be mapped using ssaha2 (false positive singletons) served as input. Mapping of the reads and SNP detection was performed by three programs: Newbler, ssahaSNP, and bwa/SAMtools. For the latter two, the duplicates were removed using cd-hit-454. The workflow resulted in a set of SNPS, whereby those found by all tools are probable the most reliable SNPS. nucleotide as well as the variant nucleotide were confirmed by at least three reads each. Hence, the minimum coverage per SNP position was six reads.

GSMapper v2.6 was run with default parameters for cDNA libraries. We constructed a .sff-file including all assembled reads of the assembly and all singleton reads that were mapped to the isotigs with ssaha2 (false positive singletons). All reads of that .sff-file were mapped against the reference sequences with GSMapper. The resulting file was parsed for SNPs using a custom script.

ssahaSNP v2.5.3 does not handle duplicate reads internally like GSMapper. Therefore, duplicate sequences were removed from the set of all assembled reads and all false positive singletons using cd-hit-454 v3.1.2 [63]. A .fastqfile was produced using the corresponding fasta and quality files of the non-duplicate sequences. ssahaSNP was run with default parameters and mapped the reads of the .fastq-file against the PUTs. The results were further processed using the parse_SNP tool provided within the ssahaSNP package and custom scripts to extract SNPs that matched our criteria.

The third approach combined two tools, bwa v0.5.9 for mapping and SAMtools v0.1.16 for variant calling. The same .fastq-file that was used for ssahaSNP was used as input for bwa. As recommended for 454 reads, the bwasw option of bwa was used. The reads were mapped against the sequences of the PUTs. SAMtools was applied to convert the resulting .sam-file to a sorted bam-file and to call the variants in that .bam-file. The resulting SNPs were again parsed to report only those SNPs with at least three reads confirming the variant and at least three reads confirming the reference nucleotide. The final set of potentially most reliable SNPs was obtained by combining the results of the three approaches and extracting those SNPs that were detected by all three programs.

Synonymous and non-synonymous polymorphisms were detected using the results of the BLASTX search of the assembly against NCBI's non-redundant $n r$ database (see below). All high-scoring segment pairs (HSP) of the top hit of each query were considered. Using the information of the BLAST results, we examined whether a SNP was in a coding or non-coding region of a gene. For SNPs in coding regions, we determined the amino acid at the corresponding position with the reference nucleotide as well as with the variant nucleotide to call synonymous or non-synonymous SNPs.

According to the criteria for SNP detection, a transcript was covered by at least six reads at each SNP position and at least three reads had to confirm each allele of a SNP. Each of the two alleles of a biallelic SNP can therefore include reads from coastal or interior varieties only, or from both varieties, resulting in nine combinations, which are summarized in Table 5 . Since there is no reference genome sequence of Douglas-fir available, it was not 
possible to decide which of the two nucleotides was the reference or the variant nucleotide. Therefore, we pooled some combinations to compare the results independently of the classification of a nucleotide as reference or variant in our results.

\section{BLAST searches and annotation}

To investigate the evolutionary conservation of the transcripts, we constructed two databases: one containing Picea sitchensis protein sequences downloaded from the NCBI data repository (picea database [64]) and one containing Arabidopsis thaliana sequences downloaded from TAIR (ara database [65]). The picea database consisted of 18,816 and the ara database of 35,381 sequences. PUTs were blasted against those two databases as well as against NCBI's non-redundant $n r$ database using BLASTX v2.2.25+ with an e-value threshold of $e^{-10}$.

Results of the BLASTX search of PUTs against $n r$ database were used as input for Blast2GO v2.4.9 [66]. Blast2GO was utilized for the functional annotation with gene ontology (GO) terms. The first step in Blast2GO was the mapping, in which GO terms associated with the hits obtained during the BLASTX search were retrieved. In the annotation step, functional terms were assigned to the sequences based on the retrieved set of GO terms per sequence using Blast2GO's annotation score. Furthermore, we used a local version of InterProScan [67] (version 4.8) to search protein signatures in the InterPro database [68]. With the local version it was possible to analyze nucleotide sequences in all six possible open reading frames. Due to the long running time of some of the InterProScan applications, we used only a subset of them that included blastprodom, fprintscan, hmmpfam, hmmpanther, hmmtigr, hmmsmart, patternscan, and seg [68]. The results of the InterProScan were imported into Blast2GO to improve annotations. Annotations were further refined using Annex and GO-Slim, both of which were available within Blast2GO [69,70]. Annex augments annotations by finding relationships between different $\mathrm{GO}$ terms and adding implicit annotations. GO-Slim represents a reduced set of $\mathrm{GO}$ terms that gives a useful summary of the all GO terms. Blast2GO provides organism specific GO-Slim mappings of which the plant specific mapping was chosen. For a better comparison of GO terms, functional annotations were generated for the protein sequences of $P$. sitchensis and $A$. thaliana used in the picea and ara databases. A BLASTP (v2.2.25+) search with an e-value of $e^{-5}$ against NCBI's non-redundant protein sequences was done before running Blast2GO. We did not annotate these two data sets with InterProScan, but with Annex and GO-Slim. The results of functional annotation of PUTs were compared to the results of the functional annotation of $P$. sitchensis and A. thaliana.

\section{Identification of drought stress related genes}

Two approaches were used to identify potential drought stress related genes. In the first approach, we divided the non-singleton PUTs, i.e. the isotigs, of the assembly by the origin of their reads into seven groups. The groups were named according to the libraries from which the reads were derived ( $c, m, s, c m, c s, m s, c m s$, where $c$ stands for control, $m$ for mild stress, and $s$ for severe stress, $\mathrm{cm}$ for control and mild stress etc.). The isotigs in the $c, m$, and $s$ groups were assumed to contain most likely treatmentspecific sequences, as they contained isotigs composed of only reads of one treatment. Therefore, we expected to find drought stress related sequences mainly in the $m$ and $s$, but also in the $m s$ groups. For the second approach, the BLASTX results were searched for specific keywords to identify candidate genes previously assigned to drought, water stress, or other stress related pathways (Table 3) [71-73].

\section{Data availability}

The sequence reads were submitted to the ENA Sequence Read Archive (SRA) under study accession number ERP001358 (http://www.ebi.ac.uk/ena/data/ view/ERP001358). PUTs, annotated SNPs, and Blast2GO results will be available from http://www.treeversity.org. BLASTX results and Python scripts used for the analysis are available upon request.

\section{Additional files}

Additional file 1: Characteristics of the libraries. Number of reads and average read length per library before and after the pre-processing steps.

Additional file 2: Read composition of the assembly. The origin as well as the number of reads assembled or otherwise marked by Newbler is illustrated.

Additional file 3: Log-log plot of assembled reads versus the sequence length. The log-log plot shows that the sequence length is depending on the number of reads assembled to the sequence.

Additional file 4: Number of isotigs per sequence length. Number of isotigs per sequence length. Reads of all twelve cDNA libraries were assembled using Newbler.

Additional file 5: Comparison of the GO-Slim categories level 3 - 5. Comparison of the distribution of the GO-Slim categories of the Douglas-fir PUTs set versus Picea sitchensis and Arabidopsis thaliana at GO level 3 to 5 . Transcriptome data of $P$. sitchensis and $A$. thaliana were obtained from NCBI and TAIR databases, respectively (See text for details).

Additional file 6: Number of identical BLASTX hits of different combination of groups after the keyword search. This table lists the number of identical BLASTX hits of different combination of groups after the keyword search. If combinations of sets are not listed, there were no equal BLASTX hits.

Additional file 7: BLASTX keyword search results. This file lists in a tab separated style for each BLASTX keyword search hit the following informations: keyword, isotig id, isotig group, hit id, hit definition, e-value. If there were more than one hit per keyword and isotig, only the best hit (i.e. the one with the smallest e-value) is listed.

Additional file 8: Schematic example of Newbler output. Schematic example of contigs, isotigs, and isogroups produced by Newbler. 
Additional file 9: Composition of the CDNA libraries in detail. Two tables describe the cDNA libraries and the provenances in detail.

\section{Abbreviations}

SNP: Single nucleotide polymorphism; s.d: standard deviation

\section{Competing interests}

The authors declare that they have no competing interests

\section{Authors' contributions}

IE designed, conducted and coordinated greenhouse and experimental work. $\mathrm{IE}$ and $\mathrm{KS}$ designed the sequencing experiment. TM analyzed the sequence data. TM, IE and KS wrote the manuscript. All authors read and approved the final manuscript.

\section{Acknowledgements}

This study is part of the collaborative project 'DougAdapt' with funding from the DFG to IE (DFG EN 829/4-1) and KS (DFG SCHM1354/3-1). The authors are grateful to Daniel Landwehr, Claudia Waack and Anna-Maria Weisser for assistance during greenhouse experiments. James Joyce, BC Timber sales, BC Randy Moench, Colorado State University Nursery, CO and Bill Taylor, Webster Forest Nursery, WA kindly provided seedlings material for this study. We are grateful to Fabian Freund for advice about statistical analysis and to Henning Wildhagen and Sariel Hübner for their feedback and suggestions on an earlier version of the manuscript. We gratefully thank the bwGRiD project (http:// www.bw-grid.de), member of the German D-Grid initiative, funded by the Ministry for Education and Research (Bundesministerium fuer Bildung und Forschung) and the Ministry for Science, Research and Arts Baden-Wuerttemberg (Ministerium fuer Wissenschaft, Forschung und Kunst Baden-Wuerttemberg) for the use of the computational resources.

\section{Author details}

${ }^{1}$ Department of Crop Biodiversity And Breeding Informatics, University of Hohenheim, Stuttgart, Germany. ${ }^{2}$ Department of Biology, University of Toronto at Mississauga, Mississauga, ON, Canada. ${ }^{3}$ Forest Research Institute of Baden-Württemberg (FVA), Freiburg i. Brsg., Germany.

Received: 26 April 2012 Accepted: 19 November 2012 Published: 28 November 2012

\section{References}

1. Hermann RK, Lavender DP: Pseudotsuga menziesii (Mirb.) Franco. In Silvics of North America: 1. Conifers. Washington, DC; 1990:527-540. Burns, RM and Honkala, BH (tech. coords.), U.S. Department of Agriculture, Agriculture Handbook 654

2. Campbell RK: Genecology of Douglas-Fir in a Watershed in the Oregon Cascades. Ecology 1979, 60(5):1036-1050.

3. Dean CA: Genotype and Population Performances and their Interactions for Growth of Coastal Douglas-Fir in Western Washington. Forest Science 2007, 53(4):463-472.

4. Rehfeldt GE: Ecological adaptations in Douglas-fir (Pseudotsuga menziesii var. glauca) populations. Heredity 1979, 43:383-397.

5. Darychuk N, Hawkins BJ, Stoehr M: Trade-offs between growth and cold and drought hardiness in submaritime Douglas-fir. Can J Forest Res 2012, 42:1530-1541.

6. Andrews SF, Flanagan LB, Sharp EJ, Cai T: Variation in water potential, hydraulic characteristics and water source use in montane Douglas-fir and lodgepole pine trees in southwestern Alberta and consequences for seasonal changes in photosynthetic capacity. Tree Physiol 2012, 32(2):146-160

7. Martinez-Meier A, Sanchez L, Dalla-Salda G, Gallo L, Pastorino M, Rozenberg P: Ring density record of phenotypic plasticity and adaptation to drought in Douglas-fir. Forest Ecol Manage 2009, 258(5):860-867.

8. Jansen K, Sohrt J, Kohnle U, Ensminger I, Gessler A: Tree ring isotopic composition, radial increment and height growth reveal provenance-specific reactions of Douglas-fir towards environmental parameters. Trees 2013, 27:37-52. [http://dx.doi.org/10. 1007/s00468-012-0765-9]
9. Carter R, Klinka K: Relationships between growing-season soil water-deficit, mineralizable soil nitrogen and site index of coastal Douglas fir. Forest Ecol Manage 1990, 30(1-4):301-311.

10. Coops NC, Coggins SB, Kurz WA: Mapping the environmental limitations to growth of coastal Douglas-fir stands on Vancouver Island, British Columbia. Tree Physiol 2007, 27(6):805-815.

11. Watkinson J, Sioson AA, Vasquez-Robinet C, Shukla M, Kumar D, Ellis M, Heath LS, Ramakrishnan N, Chevone B, Watson LT, Zyl Lv, Egertsdotter U, Sederoff RR, Grene R: Photosynthetic acclimation is reflected in specific patterns of gene expression in drought-stressed loblolly pine. Plant Physiol 2003, 133(4):1702-1716.

12. Reyer C, Lasch P, Mohren GMJ, Sterck FJ: Inter-specific competition in mixed forests of Douglas-fir (Pseudotsuga menziesii) and common beech (Fagus sylvatica) under climate change - a model-based analysis. Ann Forest Sci 2010, 67 (8):805.

13. Hermann RK, Lavender DP: Douglas-fir planted forests. New Forests 1999, 17:53-70

14. Ducić T, Parladé J, Polle A: The influence of the ectomycorrhizal fungus Rhizopogon subareolatus on growth and nutrient element localisation in two varieties of Douglas fir (Pseudotsuga menziesii var. menziesii and var. glauca) in response to manganese stress. Mycorrhiza 2008, 18(5):227-39.

15. Hanewinkel M, Cullmann DA, Schelhaas MJ, Nabuurs GJ, Zimmermann $\mathrm{NE}$ : Climate change may cause severe loss in the economic value of European forest land. Nat Clim Change 2012. [http://dx.doi.org/10.1038/ nclimate1687]

16. Intergovernmental Panel on Climate Change (IPCC): Fourth Assessment Report: Climate Change. 2007. (AR4).

17. Meehl Ga, Tebaldi $\mathrm{C}$ : More intense, more frequent, and longer lasting heat waves in the $\mathbf{2 1}$ st century. Science (New York, N.Y.) 2004 305(5686):994-7.

18. Fink $A H$, Brücher $T$, Krüger $A$, Leckebusch $G C$, Pinto JG, Ulbrich U: The 2003 European summer heatwaves and drought - synoptic diagnosis and impacts. Weather 2004, 59(8):209-216.

19. Brohan P, Kennedy JJ, Harris I, Tett SFB, Jones PD: Uncertainty estimates in regional and global observed temperature changes: $A$ new data set from 1850. J Geophys Res 2006, 111:D12106.

20. Kleinschmit J, Racz J, Weisgerber H, Dietze W, Dieterich H, Dimpflmeier R Ergebnisse aus dem internationalen Douglasien-Herkunftsversuch von 1970 in der Bundesrepublik Deutschland. Silvae genetica 1979 28(5-6):226-244.

21. Ahuja MR, Neale DB: Evolution of Genome Size in Confiers. Silvae genetica 2005, 54(3):126-137.

22. Weigel $D$, Mott $R$ : The $\mathbf{1 0 0 1}$ genomes project for Arabidopsis thaliana. Genome Biol 2009, 10(5):107.

23. Bradshaw $H$, Stettler R: Molecular genetics of growth and development in Populus. I. Triploidy in hybrid poplars. Theor App Genet 1993, 86(2-3)

24. Tuskan GA, DiFazio S, Jansson S, Bohlmann J, Grigoriev I, Hellsten U, Putnam N, Ralph S, Rombauts S, Salamov A, Schein J, Sterck L, Aerts A, Bhalerao RR, Bhalerao RP, Blaudez D, Boerjan W, Brun A, Brunner A, Busov V, Campbell M, Carlson J, Chalot M, Chapman J, Chen GL, Cooper D, Coutinho PM, Couturier J, Covert S, Cronk Q, Cunningham R, Davis J, Degroeve S, Déjardin A, dePamphilis C, Detter J, Dirks B, Dubchak I, Duplessis S, Ehlting J, Ellis B, Gendler K, Goodstein D, Gribskov M, Grimwood J, Groover A, Gunter L, Hamberger B, Heinze B, Helariutta Y, Henrissat B, Holligan D, Holt R, Huang W, Islam-Faridi N, Jones S, Jones-Rhoades M, Jorgensen R, Joshi C, Kangasjärvi J, Karlsson J, Kelleher C, Kirkpatrick R, Kirst M, Kohler A, Kalluri U, Larimer F, Leebens-Mack J, Leplé JC, Locascio P, Lou Y, Lucas S, Martin F, Montanini B, Napoli C, Nelson DR, Nelson C, Nieminen K, Nilsson O, Pereda V, Peter G, Philippe R, Pilate G, Poliakov A, Razumovskaya J, Richardson P, Rinaldi C, Ritland K, Rouzé P, Ryaboy D, Schmutz J, Schrader J, Segerman B, Shin H, Siddiqui A, Sterky F, Terry A, Tsai CJ, Uberbacher E, Unneberg P, Vahala J, Wall K, Wessler S, Yang G, Yin T, Douglas C, Marra M, Sandberg G, Van de Peer Y, Rokhsar D: The Genome of Black Cottonwood, Populus trichocarpa (Torr. \& Gray). Science 2006, 313(5793):1596-1604

25. Novaes E, Drost DR, Farmerie WG, Pappas GJJr, Grattapaglia D, Sederoff RR, Kirst M: High-throughput gene and SNP discovery in Eucalyptus grandis, an uncharacterized genome. BMC Genomics 2008, 9:312. 
26. Parchman T, Geist K, Grahnen J, Benkman C, Buerkle CA: Transcriptome sequencing in an ecologically important tree species: assembly, annotation, and marker discovery. BMC Genomics 2010, 11:180+.

27. Pauchet $Y$, Wilkinson $P$, van Munster $M$, Augustin S, Pauron D, ffrench Constant, $\mathrm{R} \mathrm{H}$ : Pyrosequencing of the midgut transcriptome of the poplar leaf beetle Chrysomela tremulae reveals new gene families in Coleoptera. Insect Biochem Mol Biol 2009, 39(5-6):403-413.

28. Pavy N, Parsons LS, Paule C, Mackay J, Bousquet J: Automated SNP detection from a large collection of white spruce expressed sequences: contributing factors and approaches for the categorization of SNPs. BMC Genomics 2006, 7:174.

29. González-Martínez SC, Ersoz E, Brown GR, Wheeler NC, Neale DB: DNA sequence variation and selection of tag single-nucleotide polymorphisms at candidate genes for drought-stress response in Pinus taeda L. Genetics 2006, 172(3):1915-26.

30. Hamrick JL, Godt MJ, Sherman-Broyles SL: Factors influencing levels of genetic diversity in woody plant species. New Forests 1992, 6:95-124.

31. Viard F, El-Kassaby YA, Ritland K: Diversity and genetic structure in populations of Pseudotsuga menziesii (Pinaceae) at chloroplast microsatellite loci. Genome 2001, 44(3):336-44.

32. Eckert AJ, Wegrzyn JL, Pande B, Jermstad KD, Lee JM, Liechty JD, Tearse $B R$, Krutovsky KV, Neale DB: Multilocus patterns of nucleotide diversity and divergence reveal positive selection at candidate genes related to cold hardiness in coastal Douglas Fir (Pseudotsuga menziesii var. menziesii). Genetics 2009, 183:289-98.

33. Meyer E, Aglyamova GV, Wang S, Buchanan-Carter J, Abrego D, Colbourne JK, Willis BL, Matz MV: Sequencing and de novo analysis of a coral larval transcriptome using $\mathbf{4 5 4}$ GSFIx. BMC Genomics 2009, 10:219.

34. Kumar S, Blaxter M: Comparing de novo assemblers for $\mathbf{4 5 4}$ transcriptome data. BMC Genomics 2010, 11:571.

35. Schuster SC, Miller W, Ratan A, Tomsho LP, Giardine B, Kasson LR, Harris RS, Petersen DC, Zhao F, Qi J, Alkan C, Kidd JM, Sun Y, Drautz DI, Bouffard P, Muzny DM, Reid JG, Nazareth LV, Wang Q, Burhans R, Riemer C, Wittekindt NE, Moorjani P, Tindall EA, Danko CG, Teo WS, Buboltz AM, Zhang Z, Ma Q, Oosthuysen A, Steenkamp AW, Oostuisen H, Venter P, Gajewski J, Zhang Y, Pugh BF, Makova KD, Nekrutenko A, Mardis ER, Patterson N, Pringle TH, Chiaromonte F, Mullikin JC, Eichler EE, Hardison RC, Gibbs RA, Harkins TT, Hayes VM: Complete Khoisan and Bantu genomes from southern Africa. Nature 2010, 463(7283):943-947.

36. Vera JC, Wheat CW, Fescemyer HW, Frilander MJ, Crawford DL, Hanski I, Marden $\mathrm{JH}$ : Rapid transcriptome characterization for a nonmodel organism using 454 pyrosequencing. Mol Ecol 2008, 17(7):1636-47.

37. Roeding F, Borner J, Kube M, Klages S, Reinhardt R, Burmester T: A 454 sequencing approach for large scale phylogenomic analysis of the common emperor scorpion (Pandinus imperator). Mol Phylogenet Evol 2009, 53(3):826-34

38. Rigault P, Boyle B, Lepage P, Cooke JEK, Bousquet J, Mackay JJ: A white spruce gene catalog for conifer genome analyses. Plant Physiol 2011 157:14-28.

39. Riggins CW, Peng Y, Stewart CNJr, Tranel PJ: Characterization of de novo transcriptome for waterhemp (Amaranthus tuberculatus) using GS-FLX 454 pyrosequencing and its application for studies of herbicide target-site genes. Pest Manag Sci 2010, 66(10):1042-52.

40. Xu L, Chen H, Hu X, Zhang R, Zhang Z, Luo ZW: Average Gene Length Is Highly Conserved in Prokaryotes and Eukaryotes and Diverges Only Between the Two Kingdoms. Mol Biol Evol 2006, 23(6):1107-1108.

41. Ashburner M, Ball CA, Blake JA, Botstein D, Butler H, Cherry JM, Davis AP, Dolinski K, Dwight SS, Eppig JT, Harris MA, Hill DP, Issel-Tarver L, Kasarskis A, Lewis S, Matese JC, Richardson JE, Ringwald M, Rubin GM, Sherlock G: Gene Ontology: tool for the unification of biology. Nat Genet 2000, 25:25-29.

42. Salem M, Rexroad CE3rd, Wang J, Thorgaard GH, Yao J: Characterization of the rainbow trout transcriptome using Sanger and 454-pyrosequencing approaches. BMC Genomics 2010, 11:564.

43. Mukhopadhyay P, Basak S, Ghosh TC: Differential Selective Constraints Shaping Codon Usage Pattern of Housekeeping and Tissue-specific Homologous Genes of Rice and Arabidopsis. DNA Res 2008, 15(6):347-356.

44. Zhang L, Li WH: Mammalian Housekeeping Genes Evolve More Slowly than Tissue-Specific Genes. Mol Biol Evol 2004, 21(2): 236-239.
45. Eckert AJ, Bower AD, Wegrzyn JL, Pande B, Jermstad KD, Krutovsky KV, St Clair JB, Neale DB: Association genetics of coastal Douglas fir (Pseudotsuga menziesii var. menziesii, Pinaceae). I. Cold-hardiness related traits. Genetics 2009, 182(4):1289-302.

46. Li P, Adams WT: Range-wide patterns of allozyme variation in Douglas-fir (Pseudotsuga menziesii). Can J Forest Res 1989, 19(2):149-161.

47. Aagaard JE, Krutovskii KV, Strauss SH: RAPDs and allozymes exhibit similar levels of diversity and differentiation among populations and races of Douglas-fir. Heredity 1998, 81:69-78.

48. Kofler R, Pandey RV, Schlötterer C: PoPoolation2: identifying differentiation between populations using sequencing of pooled DNA samples (Pool-Seq). Bioinformatics 2011, 27(24):3435-3436.

49. Hellmann I, Mang Y, Gu Z, Li P, de la Vega FM, Clark AG, Nielsen R: Population genetic analysis of shotgun assemblies of genomic sequences from multiple individuals. Genome Res 2008, 18(7):1020-1029.

50. Futschik $A$, Schlötterer $C$ : The next generation of molecular markers from massively parallel sequencing of pooled DNA samples. Genetics 2010, 186:207-18

51. Ensminger I, Schmidt L, Lloyd J: Soil temperature and intermittent frost modulate the rate of recovery of photosynthesis in Scots pine under simulated spring conditions. New Phytol 2008, 177(2):428-42.

52. Chang S, Puryear J, Cairney J: A simple and efficient method for isolating RNA from pine trees. Plant Mol Biol Reporter 1993, 11(2):113-116.

53. Zhu YY, Machleder EM, Chenchik A, Li R, Siebert PD: Reverse transcriptase template switching: a SMART approach for full-length cDNA library construction. Biotechniques 2001, 30(4):892-7.

54. Zhulidov PA, Bogdanova EA, Shcheglov AS, Vagner LL, Khaspekov GL, Kozhemyako VB, Matz MV, Meleshkevitch E, Moroz LL, Lukyanov SA, Shagin DA: Simple cDNA normalization using kamchatka crab duplex-specific nuclease. Nucleic Acids Res 2004, 32(3):e37

55. Shagin DA, Rebrikov DV, Kozhemyako VB, Altshuler IM, Shcheglov AS, Zhulidov PA, Bogdanova EA, Staroverov DB, Rasskazov VA, Lukyanov S: A novel method for SNP detection using a new duplex-specific nuclease from crab hepatopancreas. Genome Res 2002, 12(12):1935-42.

56. sff_extract. [http://bioinf.comav.upv.es/sff_extract/index.html].

57. Dlugosch KM, Rieseberg LH: SnoWhite: A pipeline for aggressive cleaning of next-generation sequence reads. [In prep]

58. SeqClean. [http://sourceforge.net/projects/seqclean/]

59. 454 Life Science: Genome Sequencer FLX System Software Manual, version 2.3; 2009 .

60. Ning Z, Cox A, Mullikin J: SSAHA: A Fast Search Method for Large DNA Databases. Genome research 2001, 11(10):1725-1729.

61. Li H, Durbin R: Fast and accurate short read alignment with Burrows-Wheeler transform. Bioinformatics 2009, 25(14):1754-60

62. Li H, Handsaker B, Wysoker A, Fennell T, Ruan J, Homer N, Marth G, Abecasis G, Durbin R, 1000 Genome Project Data Processing Subgroup: The Sequence Alignment/Map format and SAMtools. Bioinformatics 2009, 25(16):2078-9.

63. Niu B, Fu L, Sun S, Li W: Artificial and natural duplicates in pyrosequencing reads of metagenomic data. BMC Bioinformatics 2010, 11:187.

64. NCBI data repository. [http://www.ncbi.nlm.nih.gov/protein?term= picea\%20sitchensis]

65. Swarbreck D, Wilks C, Lamesch P, Berardini TZ, Garcia-Hernandez M, Foerster H, Li D, Meyer T, Muller R, Ploetz L, Radenbaugh A, Singh S, Swing $V$, Tissier C, Zhang P, Huala E: The Arabidopsis Information Resource (TAIR): gene structure and function annotation. Nucleic acids Res 2008 , 36(Database issue):D1009-14

66. Conesa A, Götz S, García-Gómez JM, Terol J, Talón M, Robles M: Blast2GO: a universal tool for annotation, visualization and analysis in functional genomics research. Bioinformatics 2005, 21(18):3674-3676.

67. Zdobnov EM, Apweiler R: InterProScan - an integration platform for the signature-recognition methods in InterPro. Bioinformatics 2001, 17(9):847-8

68. Hunter S, Apweiler R, Attwood TK, Bairoch A, Bateman A, Binns D, Bork P, Das U, Daugherty L, Duquenne L, Finn RD, Gough J, Haft D, Hulo N, Kahn D, Kelly E, Laugraud A, Letunic I, Lonsdale D, Lopez R, Madera M, Maslen J, McAnulla C, McDowall J, Mistry J, Mitchell A, Mulder N, Natale D, Orengo 
C, Quinn AF, Selengut JD, Sigrist CJa, Thimma M, Thomas PD, Valentin F, Wilson $\mathrm{D}, \mathrm{Wu} \mathrm{CH}$, Yeats $\mathrm{C}$ : InterPro: the integrative protein signature database. Nucleic acids Res 2009, 37(Database issue):D211-5.

69. Conesa A, Götz S: Blast2GO: A Comprehensive Suite for Functional Analysis in Plant Genomics. Int J Plant Genomics 2008, 2008:12.

70. Myhre S, Tveit H, Mollestad T, Lægreid A: Additional Gene Ontology structure for improved biological reasoning. Bioinformatics 2006, 22(16):2020-2027.

71. Wang W, Vinocur B, Shoseyov O, Altman A: Role of plant heat-shock proteins and molecular chaperones in the abiotic stress response. Trends in Plant Sci 2004, 9(5):244-252.

72. Shinozaki K, Yamaguchi-Shinozaki K: Gene networks involved in drought stress response and tolerance. J Exp Botany 2007, 58(2):221-227.

73. Hamanishi ET, Campbell MM: Genome-wide responses to drought in forest trees. Forestry 2011, 84(3):273-283.

doi:10.1186/1471-2164-13-673

Cite this article as: Müller et al:: A catalogue of putative unique transcripts from Douglas-fir (Pseudotsuga menziesii) based on 454 transcriptome sequencing of genetically diverse, drought stressed seedlings. $B M C$ Genomics 2012 13:673.

Submit your next manuscript to BioMed Central and take full advantage of:

- Convenient online submission

- Thorough peer review

- No space constraints or color figure charges

- Immediate publication on acceptance

- Inclusion in PubMed, CAS, Scopus and Google Scholar

- Research which is freely available for redistribution

Submit your manuscript at www.biomedcentral.com/submit

\section{( ) Biomed Central}

